\title{
Serious neutropenia following neoadjuvant chemotherapy for locally advanced breast cancer: A case report
}

\author{
ZHEN WANG, JUN-QIANG CHEN, JIN-LU LIU and XIN-GAN QIN \\ Department of Gastrointestinal Surgery, First Affiliated Hospital of Guangxi Medical University, \\ Nanning, Guangxi 530021, P.R. China
}

Received February 1, 2015; Accepted December 7, 2015

DOI: $10.3892 / \mathrm{ol} .2016 .4077$

\begin{abstract}
Neoadjuvant chemotherapy has been widely used in patients with locally advanced breast cancer (LABC) to increase the chance of breast conservation. Among the most active adjuvant chemotherapy regimens, doxorubicin and cyclophosphamide are the most common drugs used for breast cancer in adjuvant and advanced settings, and taxanes are added to neoadjuvant regimens to improve the pathological complete response rates. However, chemotherapy is often associated with a variety of acute and long-term side effects, and neutropenia is one of the most common chemotherapy-associated toxicities. Lethal neutropenia is rarely reported in clinics. The present study reports the case of a patient with LABC that received 1 cycle of neoadjuvant chemotherapy [intravenous docetaxel $\left(75 \mathrm{mg} / \mathrm{m}^{2}\right)$, pirarubicin $\left(45 \mathrm{mg} / \mathrm{m}^{2}\right)$ and cyclophosphamide $\left(500 \mathrm{mg} / \mathrm{m}^{2}\right)$ on day 2 in 3-weekly intervals] and succumbed to neutropenia and subsequent multiple organ dysfunction syndrome. The present study suggests that neutropenia may be associated with significant mortality if not managed appropriately. Based on the findings of the present study, individual chemotherapy regimens, dosing schedules, effective methods of the prevention and management of neutropenia, and the management of the discharged patient require additional consideration.
\end{abstract}

\section{Introduction}

In previous decades, the treatment of locally advanced breast cancer (LABC) has evolved considerably $(1,2)$. Initially, women with LABC were treated with radical mastectomy (3). However, the lasting effect of surgery was not completely

Correspondence to: Professor Jun-Qiang Chen, Department of Gastrointestinal Surgery, First Affiliated Hospital of Guangxi Medical University, 6 Shuangyong Road, Nanning, Guangxi 530021, P.R. China

E-mail: gxhans@163.com

Key words: breast cancer, neoadjuvant chemotherapy, neutropenia, case report satisfactory. Based on the early promising results of adjuvant chemotherapy in patients diagnosed with positive axillary lymph nodes, neoadjuvant chemotherapy was subsequently incorporated alongside surgery for the management of patients with LABC (1).

Neoadjuvant chemotherapy has several potential benefits over immediate surgery, including the eradication of possible occult distant micrometastases, the downstaging of a previously unresectable LABC to an operable tumor and decreasing the extent of surgery (3). Generally, neoadjuvant chemotherapy is indicated for patients with large breast tumors (diameter, $>5 \mathrm{~cm}$ ) that are associated with either skin or chest wall involvement (4). Currently, doxorubicin and cyclophosphamide are the most common drugs used for breast cancer in adjuvant and advanced settings, and taxanes are added to neoadjuvant regimens to improve the pathological complete response rates (2). However, these agents are often associated with a variety of acute and long-term side effects, including infusion reactions, febrile neutropenia and fatigue (5).

The present study reports the case of a patient with LABC that received docetaxel, pirarubicin and cyclophosphamide neoadjuvant chemotherapy and succumbed to neutropenia and subsequent multiple organ dysfunction syndrome. Written informed consent was obtained from the patient's family.

\section{Case report}

A 47-year-old woman was admitted to the Department of Gastrointestinal and Gland Surgery, the First Affiliated Hospital of Guangxi Medical University (Nanning, China) on July 6, 2014, due to the presence of a left breast mass for $>10$ days. The size of mass was $8 \times 7 \mathrm{~cm}$. Following admission, needle biopsy was performed: The histopathological diagnosis was infiltrating ductal carcinoma, and the tumor expressed human epidermal growth factor receptor $2 /$ neu but did not express estrogen-receptor or progesterone-receptor proteins. $\mathrm{LABC}$ is invasive breast cancer that has one or more of the following features: i) Is large (typically $>5 \mathrm{~cm}$ ); ii) has spread to several lymph nodes in the armpit (axilla) or other areas near the breast; or iii) has spread to other tissues around the breast, such as the skin, muscle or ribs. The size of the tumor in this case was $8 \times 7 \mathrm{~cm}$, therefore, the patient was diagnosed with LABC. In order to avoid skin flap grafting, the patient was advised to receive neoadjuvant 
chemotherapy prior to surgery and the patient accepted this treatment strategy.

Prior to chemotherapy, the renal, hepatic and bone marrow functions were normal. Then, docetaxel $\left(75 \mathrm{mg} / \mathrm{m}^{2}\right)$, pirarubicin $\left(45 \mathrm{mg} / \mathrm{m}^{2}\right)$ and cyclophosphamide $\left(500 \mathrm{mg} / \mathrm{m}^{2}\right)$ were administered intravenously on day 2 (TAC regimen; 3-weekly intervals). Premedication was administered in order to prevent edema, nausea and allergic reactions, including $15 \mathrm{mg}$ oral dexamethasone and $4 \mathrm{mg}$ intravenous ondansetron on days 1 , 2 and 3 of chemotherapy. Other premedications included the protection of the liver $(200 \mathrm{mg} /$ day magnesium isoglycyrrhizinate) and stomach $(80 \mathrm{mg} /$ day pantoprazole). On day 3 , routine blood and blood chemistry tests, including renal and hepatic function and electrolyte levels, were confirmed as normal. The patient felt no discomfort, and was advised to undergo routine blood tests 3-5 days subsequent to being discharged from the hospital. However, the patient did not undergo the recommended tests.

The patient was readmitted to the hospital 7 days subsequent to the chemotherapy, due to fever and abdominal pain, which she had experienced for half a day. The greatest body temperature measured $39^{\circ} \mathrm{C}$. However, no sign of peritonitis was indicated, with the exception of tenderness in the upper abdominal region. Routine blood and blood chemistry tests, including renal and hepatic function and electrolyte levels, were performed immediately. The results of the blood chemistry tests were normal, but the results of the routine blood analysis revealed that the white blood cell (WBC) count was $0.97 \times 10^{9}$ cells/l (normal range, $5.00-12.00 \times 10^{9}$ cells/1) and the absolute neutrophil count was $0.10 \times 10^{9}$ cells/ 1 (normal range, $1.80-6.30 \times 10^{9}$ cells/l). Granulocyte colony stimulating factors (G-CSFs) were administered subcutaneously and broad-spectrum intravenous antibiotics $(6 \mathrm{~g}$ /day meropenem for 2 days) were administered. However, the condition of the patient deteriorated rapidly over the next day.

The patient developed abdominal pain and breathing difficulties, and blood pressure dropped rapidly. A physical examination revealed ecchymosis in the lower back, but no sign of peritonitis. The patient was then moved to the intensive care unit (ICU) immediately. In the ICU, the patient was diagnosed with multiple organ dysfunction syndrome (MODS), and succumbed on the third day despite of active rescue. An autopsy was not performed at the request of the patient's family. The abnormal results of the auxiliary examination performed in the ICU are listed in Table I.

\section{Discussion}

Neoadjuvant chemotherapy has been widely used for patients with LABC to increase the chance of breast conservation (3). Out of the most active adjuvant chemotherapy regimens, the combination of anthracycline and taxanes is considered to be the standard preoperative treatment prior to neoadjuvant breast cancer chemotherapy (2). However, chemotherapy is often associated with a variety of acute and long-term side effects, and neutropenia is one of the most common treatment-associated toxicities (5). The cumulative risk of neutropenia is reported to range between $21-24 \%$ following adjuvant TAC chemotherapy (5). Patients that develop neutropenia experience an increased risk of serious infections and
Table I. Abnormal results of auxiliary examinations performed in the intensive care unit.

\begin{tabular}{lc}
\hline Auxiliary examination & Result \\
\hline Blood routine test, $\mathrm{n} / \mathrm{l}$ & \\
White blood cells & $0.97 \times 10^{9}$ \\
Neutrophils & $0.10 \times 10^{9}$ \\
Platelets & $34.00 \times 10^{9}$ \\
Hepatic function test & \\
Alanine aminotransferase, units/l & 4,551 \\
Aspertate aminotransferase, units/l & 11,146 \\
Cholinesterase, units/l & 2,190 \\
Total bilirubin, $\mu$ mol/l & 30.60 \\
Albumin, g/l & 15.40 \\
Coagulation function test & \\
Prothrombin time, sec & 106.80 \\
Activated partial thromboplastin & 85.90 \\
time, sec & \\
Plasma fibrinogen, g/l & 0.72 \\
Arterial blood gas analysis & \\
pH & \\
HCO ${ }_{3}^{-}$(actual bicarbonate), mmol & \\
Base excess, mmol & 5.97 \\
B-ultrasound (chest and abdomen) & $\begin{array}{l}\text { Hydrothorax and ascites, } \\
\text { and abdominocentesis }\end{array}$ \\
& revealed incoagulable \\
& blood \\
\hline & \\
\hline
\end{tabular}

often require hospitalization (5). Therefore, the prevention and management of neutropenia is an important issue in adjuvant chemotherapy of breast cancer.

The present study reported the case of a patient with LABC that received 1 cycle of TAC neoadjuvant chemotherapy and developed neutropenia. Ultimately, the patient succumbed to MODS, despite active rescue. The outcome suggests that neutropenia may be associated with mortality if not managed appropriately, although lethal neutropenia is rarely reported in the clinic. Based on the present case, several concerns may be noted, including how best to individualize chemotherapy regimens and dosing schedules. Previous studies indicate that combination chemotherapy may generate high response rates, but is associated with a higher toxicity rate and no clear survival advantage over sequential monotherapy $(6,7)$. However, sequential single-agent chemotherapy is not only associated with a reasonable chance of response, but also the successful palliation of symptoms and an improved quality of life while minimizing toxicity (6). Therefore, combination regimens are generally recommended for patients with rapidly progressive or symptomatic visceral disease, and sequential single-agent chemotherapy is generally recommended for patients with metastatic breast cancer (6). As for dosing schedules, studies report that weekly schedules may cause fewer neutropenic complications compared with 21-day schedules, but demonstrate decreased anticancer activity and increased rates of skin toxicity and fatigue (8). Therefore, the method 
for the individualization of chemotherapy regimens and dosing schedules for patients with neoadjuvant LABC remains unclear.

The best practice for rescuing patients following the development of serious neutropenia is uncertain. Previous studies indicate that the use of G-CSFs may decrease the duration of neutropenia, fever and the length time spent in hospital for chemotherapy, but do not improve the survival rate $(5,9)$. Therefore, guidelines recommend against the routine use of G-CSFs for patients with established neutropenia (9). However, it is unclear whether there may be an argument for the use of G-CSFs in conditions such as MODS. In addition, there is uncertainty as to whether the routine use of G-CSFs as a primary prophylaxis to reduce the incidence of neutropenia is necessary, and additional studies are required.

The median length of time spent in hospital for chemotherapy in China is 3 days, and long-term stays are typically not possible, unless morbidities occur. Patients that receive combination chemotherapy most commonly possess a decreased WBC count 10-14 days subsequent to initial administration (5). Therefore, ensuring the compliance of patients is a considerable problem in China. The clinical outcome may be different for future patients if the case reported in the present study is reviewed.

The present study reported the case of a patient with LABC that received neoadjuvant chemotherapy and succumbed to neutropenia and subsequent MODS. Based on the findings of the present case, individual chemotherapy regimens, dosing schedules, effective methods for the prevention and management of neutropenia, and the management of discharged patients require additional attention in future studies.

\section{References}

1. Rastogi P, Anderson SJ, Bear HD, Geyer CE, Kahlenberg MS Robidoux A, Margolese RG, Hoehn JL, Vogel VG, Dakhil SR, et al: Preoperative chemotherapy: Updates of National Surgical Adjuvant Breast and Bowel Project Protocols B-18 and B-27. J Clin Oncol 26: 778-785, 2008.

2. Gampenrieder SP, Rinnerthaler G and Greil R: Neoadjuvant chemotherapy and targeted therapy in breast cancer: Past, present, and future. J Oncol 2013: 732047, 2013.

3. Rapoport BL, Demetriou GS, Moodley SD and Benn CA: When and how do I use neoadjuvant chemotherapy for breast cancer? Curr Treat Options Oncol 15: 86-98, 2014.

4. Singletary SE, Allred C, Ashley P, Bassett LW, Berry D, Bland KI, Borgen PI, Clark G, Edge SB, Hayes DF, et al: Revision of the American Joint Committee on Cancer staging system for breast cancer. J Clin Oncol 20: 3628-3636, 2002.

5. Ho MY and Mackey JR: Presentation and management of docetaxel-related adverse effects in patients with breast cancer. Cancer Manag Res 6: 253-259, 2014.

6. Piccart-Gebhart MJ, Burzykowski T, Buyse M, Sledge G, Carmichael J, Lück HJ, Mackey JR, Nabholtz JM, Paridaens R, Biganzoli L, et al: Taxanes alone or in combination with anthracyclines as first-line therapy of patients with metastatic breast cancer. J Clin Oncol 26: 1980-1986, 2008.

7. Iwata H, Sato N, Masuda N, Nakamura S, Yamamoto N, Kuroi K, Kurosumi M, Tsuda H, Akiyama F, Ohashi Y, et al: Docetaxel followed by fluorouracil/epirubicin/cyclophosphamide as neoadjuvant chemotherapy for patients with primary breast cancer. Jpn J Clin Oncol 41: 867-875, 2011.

8. Morris PG, McArthur HL, Hudis C and Norton L: Dose-dense chemotherapy for breast cancer: What does the future hold? Future Oncol 6: 951-965, 2010.

9. Aapro MS, Bohlius J, Cameron DA, Dal Lago L, Donnelly JP, Kearney N, Lyman GH, Pettengell R, Tjan-Heijnen VC, Walewski J, et al; European Organisation for Research and Treatment of Cancer: 2010 update of EORTC guidelines for the use of granulocyte-colony stimulating factor to reduce the incidence of chemotherapy-induced febrile neutropenia in adult patients with lymphoproliferative disorders and solid tumours. Eur J Cancer 47: 8-32, 2011. 